\title{
Endoluminal Perspective Volume Rendering of Coronary Arteries Using Electron-Beam Computed Tomography
}

\author{
Nobusada Funabashi, MD; Kazuo Misumi, MD*; Hiroyuki Ohnishi, RT*; \\ Mai Watanabe, RT*; Yutaka Suzuki, RT*; Noriyuki Imai, RT*; \\ Katsuya Yoshida, MD; Issei Komuro, MD
}

\begin{abstract}
Remarkable progress has been made in the treatment of coronary heart diseases because of a variety of new interventional devices, but as each new device or procedure has suitability for a particular type of patient or purpose, patient selection is increasingly important. Endoluminal perspective volume renderings of the coronary arteries of a 70-year-old male with old myocardial infarction and recurrent chest pain were carried out using electron-beam computed tomography. Conventional coronary angiography had revealed significant stenosis of the distal portion of the left anterior descending branch, and subsequent conventional balloon angioplasty had failed to expand the stenotic site. Perspective volume rendering images can distinguish differences in objects and evaluate the cross sectional area of the lumen and the morphology of calcification. In the present patient, a huge mass of calcified plaque occupied most of the lumen at a site corresponding to the angiographic site of stenosis. According to this finding, rotational atherectomy was indicated and had a good outcome. The qualitative information for characterizing and determining the morphology of atherosclerotic plaque provided by perspective volume rendering may be useful in selecting the appropriate intervention. (Circ J 2003; 67: 1064-1067)
\end{abstract}

Key Words: Coronary arteries; Electron-beam computed tomography; Perspective volume rendering

$\mathbf{R}$ ecently it has become possible to evaluate coronary arteries using non-invasive modalities such as computed tomography (CT) or magnetic resonance imaging!,2 In addition, perspective rendering of the coronary arteries using electron-beam CT (EBCT), which has the capability of electrocardiogram triggering and has excellent temporal and spatial resolution, has been reported in conjunction with the shaded surface display technique? Qualitative information on the characterization and morphology of atherosclerotic plaque would be useful in selecting the appropriate device or procedure as intervention in coronary heart disease for individual patients. We report our experience with endoluminal perspective volume rendering (PVR) of the coronary arteries using EBCT.

\section{Case Report}

Both conventional coronary angiography and EBCT were performed on a 70-year-old male within a period of $24 \mathrm{~h}$. He had an old myocardial infarction, and balloon angioplasty without stenting had been performed 2 years previously because of stenosis of the distal portion of the left anterior descending coronary artery (LAD). However, it had failed to expand the stenotic site and the patient had experienced recurrent chest pain for 1 month prior to this study. Conventional coronary angiograms revealed signifi-

(Received April 27, 2002; revised manuscript received June 3, 2002; accepted June 10, 2002)

Department of Cardiovascular, Science and Medicine, Chiba University Graduate School of Medicine (M4) and *Heart Institute, ChibaNishi General Hospital, Chiba, Japan

Mailing address: Issei Komuro, MD, Department of Cardiovascular Science and Medicine, Chiba University Graduate School of Medicine, 1-8-1 Inohana, Chuo-ku, Chiba City, Chiba 260-8670, Japan.

E-mail: komuro-tky@umin.ac.jp

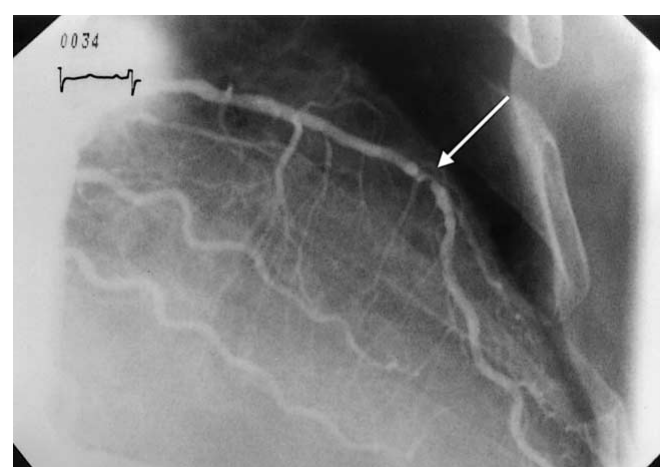

Fig 1. Conventional coronary angiogram. The right anterior oblique projection shows significant stenosis in the distal portion of the left anterior descending coronary artery (arrow).

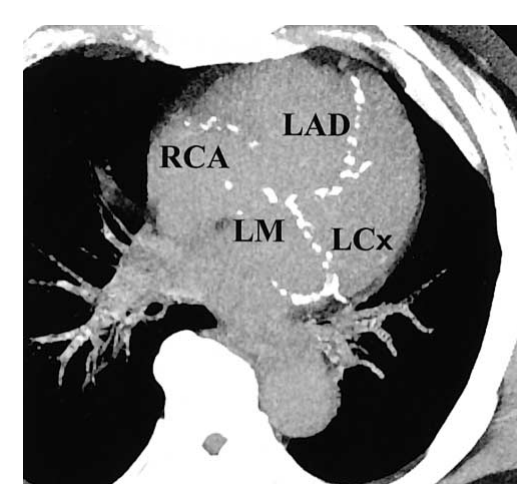

Fig 2. One thin slab maximum intensity projection image of the coronary arteries using routine electron-beam computed tomography. Much calcification is evident in the left main (LM), left anterior descending coronary artery (LAD), left circumflex branch (LCx), and right coronary arteries (RCA). 


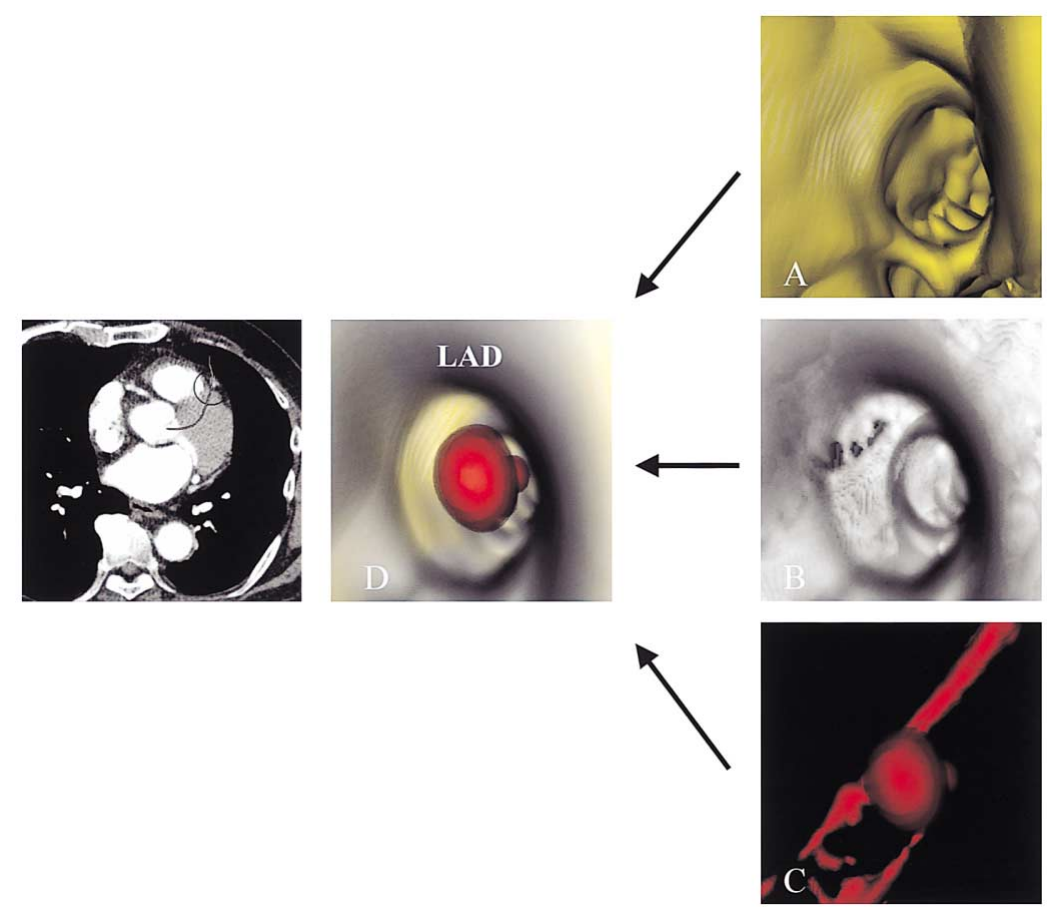

Fig 3. Schema of the concept of endoluminal perspective volume rendering (PVR) images. Axial source image of enhanced electron-beam computed tomography (EBCT) and endoluminal PVR images of the coronary arteries using EBCT. The curved line and circle in the axial source image show the route of the series of endoluminal PVR images and the site of the endoluminal PVR images in (A-D), respectively. The endoluminal PVR images show the inside of the lumen of the coronary arteries. All PVR images (A-D) were of the same view and site, but with different shapes of opacity and color curves. (A) The shape of the opacity curves was set to eliminate visualization of the calcification, contrast medium from the lumen, and vessel wall, and was set to represent fatty tissue. As a result, epicardial fat was represented. The shape of the color curves was set to represent the fatty tissue as yellow. (B) The shape of the opacity curves was set to eliminate visualization of the calcifications, contrast medium from the lumen, and epicardial fat, and was set to represent the vessel wall as semitransparent. The shape of the color curves was set to represent the vessel wall as white. (C) The shape of the opacity curves was set to eliminate visualization of the contrast medium from the lumen, vessel wall and fatty tissue, and was set to represent calcified plaque with CT numbers well above those of the contrast-enhanced coronary artery lumen. The shape of the color curves was set to represent calcified plaque as red. (D) The shapes of the opacity and color curves were combined with those of A, B, and C. Epicardial fat could be observed through the semitransparent vessel wall. The form of the calcified plaque and the spatial relationship of the calcified plaque, vessel wall, and epicardial fat could be recognized.

cant stenosis of the distal portion of the LAD (Fig 1).

Next day, a routine EBCT scan (Imatron C-150XP, Imatron, South San Francisco, CA, USA) was performed to evaluate calcification of the coronary arteries. While the patient held his breath, the scan was performed in the step volume scan (SVS) mode using $3 \mathrm{~mm}$ collimation, $3 \mathrm{~mm}$ table incrementation, $100 \mathrm{~ms}$ scanning time and triggered to $80 \%$ of the R-to-R interval. The thin slab maximum intensity projection image indicated severe calcification on the left main, LAD, left circumflex branch, and right coronary arteries (Fig 2). Next, to evaluate the coronary arterial lumen and to make endoluminal PVR, enhanced EBCT was performed using the SVS mode with $3 \mathrm{~mm}$ collimation and $2 \mathrm{~mm}$ table incrementation and was coupled to a $150 \mathrm{ml}$ intravenous injection of iodinated contrast medium $(300 \mathrm{mgI} / \mathrm{ml})$ at $2.5 \mathrm{ml} / \mathrm{s}$ with a delay time of $25 \mathrm{~s}$. These data were sent to a workstation (M-900, Zio, Tokyo, Japan) and 3-dimensional (D) images were reconstructed. The endoluminal PVR images showed the inside of the lumen of the coronary arteries and we could represent the structure surrounding the lumen (vessel walls and fatty tissue) and the calcification by selecting the appropriate shape of the opacity and color curves. The shape of the opacity curves was set to eliminate visualization of the contrast medium from the lumen to show the inside of the vessel lumen, and was set as semitransparent to represent the vessel walls. Fatty tissue and calcifications were represented as completely opaque. The shape of the color curves was set to represent fatty tissue, vessel walls, and calcifications as yellow, white, and red, respectively (Fig 3). Calcified plaque with CT numbers well above those of the contrastenhanced coronary artery lumen could be distinguished by PVR. There was a huge mass of calcified plaque occupying most of the lumen at a site approximately equal to the previously identified site of stenosis (Fig 4E,F), so high-speed rotational atherectomy of the LAD was selected as the interventional procedure and had a good result.

\section{Discussion}

EBCT obtains ultrafast scans by sweeping a steered electron beam on a fixed tungsten target ring, thereby providing a moving X-ray source without mechanical motion. The 100 -ms mode is used for high-resolution cross-sectional imaging in the SVS mode with ECG gating. Therefore, EBCT is a suitable modality for cardiac imaging because of its high spatial resolution and the ability to perform ECG triggering. The spatial resolution for in-plane in EBCT is 


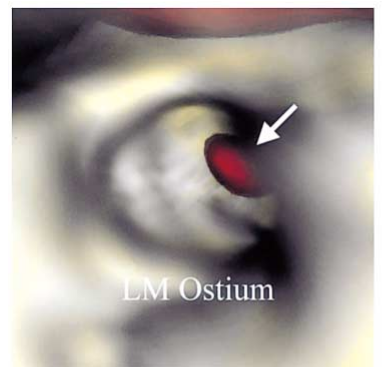

A

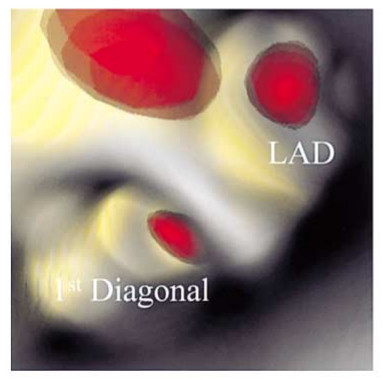

C

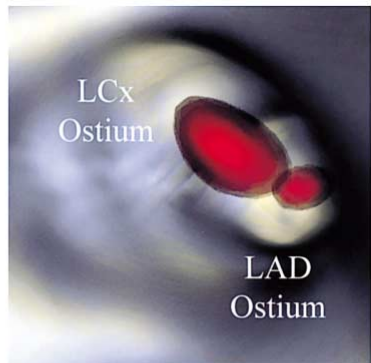

B

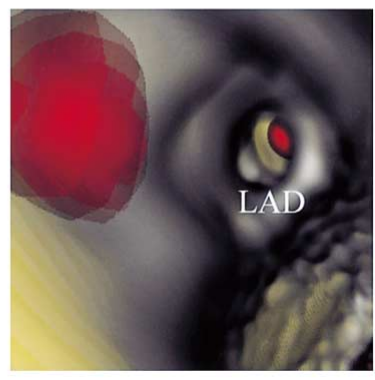

D

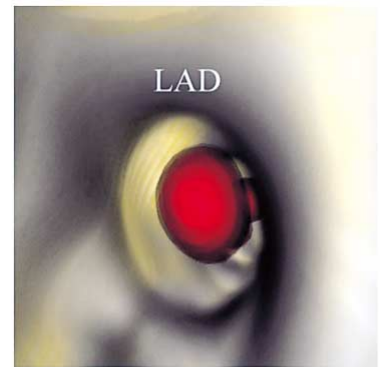

$\mathbf{E}$

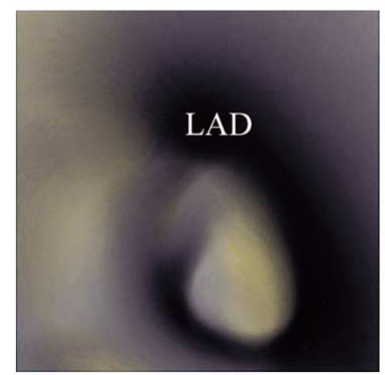

G

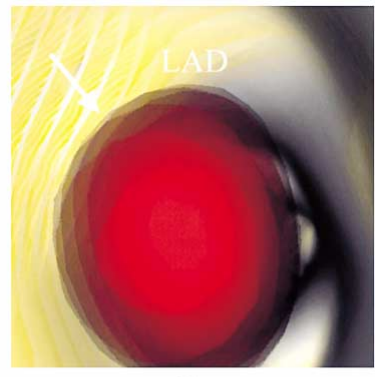

$\mathbf{F}$

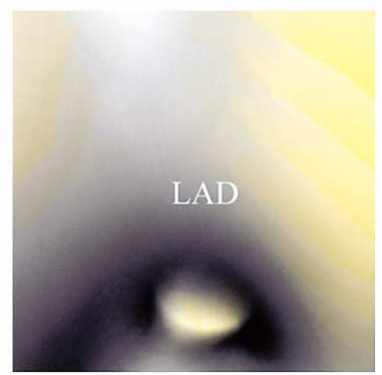

H

Fig 4. Endoluminal perspective volume renderings of the coronary arteries using electron-beam computed tomography (EBCT) show the inside of the lumen of the coronary arteries. The shapes of the opacity curves and color curves were the same as in Fig 3D. Calcified plaque with CT numbers well above those of the contrast-enhanced coronary artery lumen could be distinguished by perspective volume rendering. (A) The left main (LM) ostium is shown. Calcified plaque as represented in red is inside the LM (arrow). (B) Bifurcation of the left anterior descending coronary artery (LAD) and the left circumflex branch (LCx) is shown. Calcified plaque in the bifurcation of the LAD artery and LCx and LAD ostium can be noted. (C) Bifurcation of the LAD and the first diagonal branch (1st Diagonal) is shown. (D) The lumen of the LAD is shown. The right lower part of the vessel wall was eliminated by an inappropriate setting of the opacity curves artificially. (E,F) The lumen of the distal part of the LAD is evident. There was a huge mass of calcified plaque (arrow) and that site was approximately equal to the corresponding site of stenosis as revealed by conventional coronary angiograms. $(\mathrm{G}, \mathrm{H})$ The lumen of the further distal part of the LAD is shown. Calcified plaque was not observed, possibly because of the limits of capability of the spatial resolution of the EBCT scanner and partial volume effects.

approximately $0.71 \mathrm{~mm}$.

Perspective rendering of the colon, bronchus, and aorta using helical CT data has been reported ${ }^{4,5}$ and its advantage is that it is non-invasive compared with endoscopy and intravascular ultrasound (IVUS). In addition, the distal site can be observed by virtual passage through the stenotic site, which is difficult using real endoscopy, and after acquisition, the images can be reviewed from any direction desired.

With conventional angiograms and 3-D images obtained by $\mathrm{CT}$ and magnetic resonance angiography, the vessel lumen is observed from the outside and redundant objects, such as bone, vessels, internal organs, and muscles, must be eliminated. For coronary arteries, for example, this can take approximately $20 \mathrm{~min}$ even by experienced technicians; more commonly it takes approximately $1 \mathrm{~h}$ ? Such timeconsuming elimination of redundant objects is unnecessary with PVR because it observes the target subject from the inside as a cross sectional area.

One problem with 3-D images using CT and magnetic resonance image data is that if a fixed threshold is adopted the vessel diameter or the cross-sectional area of one part may be overestimated and those of another part underestimated because of differences in the degree of enhancement during imaging, in addition to the partial volume effect? Adoption of a segmented threshold using line density profile curves can provide more accurate results than the use of a fixed threshold? To make endoluminal PVR of coronary arteries using EBCT, we can set any threshold or opacity and color curve for each key frame to achieve a segmented threshold or opacity and color curves, but this methodology cannot provide information on the essential color of an atherosclerotic atheroma, such as white or yellow.

Much of the remarkable progress in the treatment of coronary heart diseases has been through advances in percutaneous transluminal coronary angioplasty, especially with regard to the variety of new interventional devices. Each new device or procedure has suitability for a particular type of patient or purpose, so patient selection is very important. Calcification is a principal factor in determining the intervention; for example, suitable sites for rotational atherectomy are those that have extensive calcification, 10 diffuse lesions, ostial lesions, or lesions that are difficult to expand.

Endoluminal PVR of coronary arteries using EBCT makes it possible to distinguish differences in objects, such as calcification, smooth muscle cells, and fatty tissue!1 Compared with axial source images obtained by routine EBCT scanning, which has been the gold standard for detecting and quantifying coronary arterial calcification, ${ }^{12}$ PVR images enable the clinician to evaluate the configuration of the calcification against the cross-section of the vessel lumen; for example, how closely the angle of the calcium against the lumen reaches $360^{\circ}$ or whether the calcification is eccentric. Thus, this information, previously only available from IVUS, can be obtained non-invasively and support the selection of the appropriate intervention. A case-control study need to be performed to determine the 
usefulness of PVR as a tool in selecting the appropriate intervention for coronary heart disease, and should include a comparison with the findings of IVUS.

\section{References}

1. Moshage WE, Achenbach S, Seese B, Bachmann K, Kirchgeorg M. Coronary artery stenoses: Three-dimensional imaging with electrocardiographically triggered, contrast agent-enhanced, electron beam CT. Radiology 1995; 196: 707-714.

2. Shimamoto R, Suzuki J, Nishikawa J, Fujimori Y, Nakamura F, Shin WS, et al. Measuring the diameter of coronary arteries on MR angiograms using spatial profile curves. AJR Am J Roentgenol 1998; 170: 889-893.

3. Nakanishi T, Kohata M, Miyasaka K, Fukuoka H, Ito K, Imazu M. Virtual endoscopy of coronary arteries using contrast-enhanced ECG triggered electron beam CT data sets. AJR Am J Roentgenol 2000; 174: $1345-1347$.

4. Beaulieu CF, Jefferey RB Jr, Karadi C, Paik DS, Napel S. Display modes for CT colonography: Part II. Blinded comparison of axial CT and virtual endoscopic and panoramic endoscopic volume-rendered studies 1. Radiology 1999; 212: 203-212.

5. Neri E, Caramella D, Falaschi F, Sbragia P, Vignali C, Laiolo E, et al. Virtual CT intravascular endoscopy of the aorta: Pierced surface and floating shape thresholding artifacts 1. Radiology 1999; 212: 276 279.
6. Rubin GD, Beaulieu CF, Argiro V, Ringl H, Norbash AM, Feller JF, et al. Perspective volume rendering of CT and MR images: Applications for endoscopic imaging. Radiology 1996; 199: 321-330.

7. Achenbach S, Moshage W, Ropers D, Nossen J, Daniel WG. Value of electron-beam computed tomography for the noninvasive detection of high-grade coronary-artery stenoses and occlusions. $N$ Engl J Med 1998; 339: 1964-1971.

8. Achenbach S, Moshage W, Ropers D, Bachmann K. Comparison of vessel diameters in electron beam tomography and quantitative coronary angiography. Int J Card Imaging 1998; 14: 1-7.

9. Funabashi N, Rubin GD, Kobayashi Y, Shifrin RY, Wexler L, Perlroth M. Accuracy of coronary artery dimensions with ElectronBeam CT angiography: Comparison of measurement methods. Radiology 1999; 213(P): 269.

10. Brown DL, George CJ, Steenkiste AR, Cowley MJ, Leon MB, Cleman MW, et al. High-speed rotational atherectomy of human coronary stenoses: Acute and one-year outcomes from the new approaches to coronary intervention (NACI) registry. Am J Cardiol 1997; 80: 60K-67K

11. Funabashi N, Matsumoto A, Yoshida T, Watanabe S, Misumi K, Masuda Y. Usefulness of three-dimensional visualization of coronary arteries using electron-beam computed tomography data with volume rendering. Jpn Circ J 2000; 64: 644-646.

12. Agatson AS, Janowitz WR, Hildner FJ, Zusmer NR Jr, Viamonte M, Detrano R. Quantification of coronary artery calcium using ultrafast computed tomography. J Am Coll Cardiol 1990; 15: 827-832. 